\title{
Empirical Performance of Option Pricing Models: Evidence from India
}

\author{
Vipul Kumar Singh
}

Correspondence: Vipul Kumar Singh, Institute of Management Technology (IMT), $35 \mathrm{Km}$ Milestone, Katol Road, Nagpur - 441502, India. Tel: 91-712-280-5138. E-mail: vksing@imtnag.ac.in

Received: October 10, 2010

Accepted: December 27, 2012

Online Published: January 11, 2013

doi:10.5539/ijef.v5n2p141

URL: http://dx.doi.org/10.5539/ijef.v5n2p141

\begin{abstract}
This paper empirically investigates the comparative competitiveness of the family of option pricing models categorized as deterministic and stochastic. Forecasting effectiveness of the models is judged on the basis of pricing accuracy of the models. For same this paper categorically examine the out-of-sample moneyness-maturity forecasting performance of models. Data set of Nifty index options of India is especially chosen for analyzing the effectiveness of models. Pricing imperfections of models is compare and contrasted with the market price of the options. Cross competitiveness of the models is empirically testifies with the benchmark Black-Scholes but relative to market using well-known technique of error metrics. Expected price of the models inferred analytically by estimating the parameters of the models continuously, almost every day. The models are inter-pass through the recent waves of financial upheavals and has been put into a practical implication of fastest descending movement of Indian capital market. We found that the Practitioner Black-Scholes and Heston model has smaller out of sample valuation errors in pricing Nifty Index options than the Constant Elasticity of Variance, Gram-Charlier, and Hull \& Whit models, but no models eliminates price bias completely.
\end{abstract}

Keywords: black-scholes, call options, deterministic, implied volatility, nifty index, stochastic

JEL: C01, C13, C52, C53, G17

\section{Introduction}

The history of option pricing is century old, dates back to 1900 when the French mathematician Louis Bachelier (1900) first applied the concept of Brownian motion in pricing of stocks. Over the years, his conceptual framework becomes the norm in mathematical finance. Initially the option pricing was deriving by taking the discounted expectation of underlying payoff until its maturity. In 1973, Fisher Black, Maryon Scholes and Robert Merton provided major breakthrough and discovered the formula that revolutionized the pricing and trading of options. The pricing framework of trio also set the new benchmark in the history of mathematical finance and set the foundation of new area widely known as financial engineering.

Though the BS formula become the prime tool for pricing options but its empirical deficiencies (BS model shows systematic price bias across moneyness and maturity) provoked researchers for the development of advance models (Rubinstein, 1985; Hull and White, 1987; Wiggins, 1987; Dumas, Fleming and Whaley, 1998). The flock reveals that the two unrealistic assumptions of the model: the asset return follow log-normal distribution and volatility of the underlying remains constant throughout the life of the options is mainly responsible for deviation between model and market (Cont, 2001). The pricing deficiency of the BS model can also be depict by plotting a graph between following three: BS implied volatilities, strike price (X) or moneyness $(\mathrm{X} / \mathrm{S})$ and time to expiry. Instead of a neutral facial expression, the plot exhibits a parabolic shape, skewed largely to out-of-the-money and higher maturity options. The plot is widely known as the volatility smile/smirk pattern. Rubinstein (1994) and Heynen (1993) examine the same for the S\&P 500 index and European Options Exchange.

Together, the theoretical and practical underpinnings of BS model induce researchers to pursuit the development of more realistic models, incorporating non-lognormal and stochastic features of the stock price and its volatilities. In the past four decades, researchers have suggested numerous models to price options with non-constant asset price volatility (Abken and Nandi, 1996). The proposed models divided in two veins: deterministic and stochastic volatility models (Ball and Roma, 1994). Deterministic volatility models are based on the framework that volatility is determined by some variables observable in the market whereas stochastic 
volatility models are based on the framework that volatility is itself stochastic whose parameters are not directly observable in the market (Stein and Stein, 1991). Though several models has been suggested but only few managed to gain the popularity and retain the attention of practitioners. In stochastic family, model of Hull \& White (1987), Heston (1993) and Heston and Nandi GARCH (2000) are the most popular while in deterministic category Constant Elasticity of Variance (CEV) model of Cox (1976), Deterministic Volatility Functions (DVF's) of Dumas, Fleming and Whaley (1998) and Gram-Charlier model of Backus, Foresi, and Wu (2004) managed to gain the popularity. Deterministic CEV expressed the volatility as a function of the price of the underlying asset, DVF modeled parabolic shape of the volatility smile and Gram-Charlier model incorporated excess skewness and kurtosis. Stochastic HW modeled the correlation of underlying return and volatility, and in addition Heston's (1993) focuses on non-lognormal distribution of the assets return, leverage effect and mean-reverting property of volatility.

Nifty index option is the most traded instrument on the browse of National Stock Exchange (NSE) of India and accounts more than $75 \%$ trade of the NSE alone. Due to Nifty index options NSE manage to rank among the top five exchanges of the world. In order to price Nifty index options this research work focuses on the entwined relation of option pricing and volatility of the underlying instrument (Scott, 1987). Like other stock exchanges, National Stock Exchange (NSE) of India is also using the BS model as a benchmark tool to fix the base prices of options underlying Nifty index and stocks, despite of its shortcomings. Therefore, this paper focuses on the empirical effectiveness of deterministic and stochastic models and tries to testify their applicability relative to market prices. This work is an extension of the empirical research work of Brockman and Chowdhury (1997) and explores the effectiveness of models of deterministic and stochastic families in the current scenario of financial upheavals.

To testify the comparative competitiveness of option pricing models we imposed four restrictions on the data set employed. The first one is instead of intra-daily closing prices are used. This restriction should however not affect the results in a significant way since Nifty index options is highly liquid implying that the closing prices of the options and the stock index is reasonably synchronous. The second restriction is regarding the estimation of models parameters and uses of numerical technique of non-linear least square for same as the method requires the simultaneous uses of market and model option prices. The third restriction is that dividends are not taken into account. This should in general not have any significant effects on the results since many of the stocks in the Nifty 50 index pay dividends only once or twice in a year. The fourth and final restriction is that only call options are valued. However, utilizing put-call parity put options can also be easily valued using the put-call parity.

In this research, models will be simulated using Excel VBA and Eviews. We will specifically look at the relative errors produced by the model prices with respect to the market prices. By comparing the relative errors, we expect to find the best model that can fully describe the market. The objective of this research is to discuss empirical techniques employed in testing option-pricing models, and to summarize major conclusions from the empirical literature.

The rest of the paper is organized as follows. Section 2 describes the data. Section 3 presents the relevant deterministic and stochastic volatility models. Section 4 provides the process of estimation of parameters of models. Section 5 compare and contrast the results of the models, besides that the section also briefly discussed the pattern of implied volatility exhibited by models across moneyness and maturity. Section 6 finally concludes our study

\section{Data Description}

This research work requires the collection of historical data of models parameters namely index price, strike price, time to maturity and risk free rate of interest. Except risk free rate of interest, all others are readily available from the official website of NSE. Since, there is direct data matching of risk free interest rate for the purpose of this research we utilized the yield of T-bill of 91-day duration issued by Reserve Bank of India (RBI) considered as risk free. The data is collected from the electronic database of the RBI.

\subsection{Data Screening Procedure}

Before making the real applicability of call options data collected from the bourse of NSE, all option data set is first inter-pass through following four series of exclusionary filters. First, option data set checked for lower boundary condition

$$
S_{t}-K e^{-r t} \leq C\left(S_{t}, t\right)
$$


where $S_{t}$ is the current asset price, $K$ is the strike price, $r$ is the risk-free interest rate, and $C\left(S_{t}, t\right)$ is the call price at time $t$. Call option data set not satisfying the lower boundary condition is considered as an invalid observation and thus plucked out. Second, since very deep out-of-the-money and very deep in-the- money options are not traded actively on NSE and their price quotes generally not reflect the true option value hence data of moneyness greater (less) than $15 \%(-15 \%)$ is excluded. We found that option with maturities greater than 90 days is very less actively traded on the bourse of NSE thus rejected. Since options with less than five days of maturity are highly sensitive to price-volatility bias, we discarded them also from the database. The final set of remaining data is figured into 7455 call option.

\subsection{Option Categories}

The filtered set of data is then arranged categorically moneyness-maturity wise in fifteen categories: five moneyness and three maturities. Each market option data is placed in one of the fifteen categories depending on their time to expiration and ratio of the asset price to the strike price. Three ranges of time to expiration are distinguished as short maturity (0-30 days or below one month), medium maturity (30 to 60 days or between one to two months), long maturity (60 to 90 days or between two to three months). Since option prices are very sensitive to their exercise price and time to maturity, for the purpose of this research we divided the option data into five categories of moneyness: at-the-money (ATM) if the moneyness is in between $(-5 \%, 5 \%)$, in-the-money (ITM) if the moneyness is in between $(5 \%, 10 \%)$, out-of-the-money (OTM) if the moneyness is in between $(-10 \%,-5 \%)$ and deep in-the-money (DITM) if the moneyness is greater than $10 \%$ and deep out-of-money (DOTM) if the moneyness is less than $-10 \%$.

\subsection{Methodology}

Since volatility is the most crucial and only unknown parameter in the classical BS model; the implied volatility inferred reciprocally from the BS can also be used to justify the accuracy of the BS and other related option-pricing models. For same, the out-of sample forecasting performance of the models has been compare and contrasted. In order to have sustainable input parameters, models parameters is computed analytically by optimization techniques (Coleman and $\mathrm{Li}, 1996)$ which further use as an input to figure out the effectiveness of the models against benchmark BS model for pricing Nifty index option contract with market value. This paper adopted an effective statistical tool for evaluating the performance of option pricing models that involves calculating the error metrics. To see how well a model performs, we look at the relative error generated by the models.

To provide a distinctive framework instead of comparing the results of the models with the classical BS we have compared them with market and checked the competitiveness of the models relative to market. For same we utilized the two well-known error metrics, percentage mean error $(P M E)$ and mean absolute pricing error (MAPE), described as

Percentage Mean Error (PME)

$$
P M E=\frac{1}{K} \sum_{i=1}^{K}\left(C_{i}^{\text {Model }}-C_{i}^{\text {Market }}\right) / C_{i}^{\text {Market }}
$$

Mean Absolute Pricing Error (MAPE)

$$
\text { MAPE } \left.=\text { Mean } \quad\left[C_{i}^{\text {Model }}-C_{i}^{\text {Market }}\right\rceil\right]
$$

where $C_{i}^{\text {Model }}$ is the expected price and $C_{i}^{\text {Market }}$ is the actual price of the call option of the ith observation and $k$ is the number of total observations. The sign and magnitude of the relative error of PME and MAPE will decide the competency of the models. In case of PME, the sign and magnitude together will decide the quality of the model. A large negative (positive) relative PME would mean that the model under prices (overprices) the specific option whereas in case of MAPE magnitude will decide the degree of overpricing and under pricing i.e. good or bad approximation to the market.

\section{Option Pricing Models}

\subsection{The Black-Scholes Option Pricing Model}

The model hardly requires any introduction, due to its simplicity, closed-form solution, and ease of implementation it is the most popular option-pricing model. The BS option pricing formula for a stock paying no dividend is 


$$
C_{B S}=S N\left(d_{1}\right)-K e^{-r t} N\left(d_{2}\right)
$$

where

$$
\begin{gathered}
d_{1}=\frac{\ln [S / K]+\left[r+0.5 \sigma^{2}\right] t}{\sigma \sqrt{t}} \\
d_{2}=\frac{\ln [S / K]+\left[r-0.5 \sigma^{2}\right] t}{\sigma \sqrt{t}}=d_{1}-\sigma \sqrt{t}
\end{gathered}
$$

$C$ denotes the price of a call option, $S$ denotes the underlying Index price, $K$ denotes the option exercise price, $t$ is the time to expiry in years, $r$ is the risk free rate of return, $N(d)$ is the standard normal distribution function, and $\sigma^{2}$ is the variance of returns on the Index. The important specification of the formula is that it can also be used to price variety of options underlying different assets class with little modification in the base framework.

\subsection{The Practitioner Black-scholes Model}

The ample of empirical evidences proving the existence of parabolic smile/skew shape of the implied volatility and its serial dependence on moneyness and maturity, violating the non-constant volatility assumption of BS had motivated practitioners to explore the dependence of implied volatility on moneyness and maturity. Dumas, Fleming, and Whaley (1998) modeled that as the liner quadratic function of three. Researchers named it deterministic volatility functions (DVF's). For the purpose of this research, we focused on only following three specifications of DVF:

$D V F 1$

$$
\sigma_{i v}=a_{0}+a_{1} K+a_{2} K^{2}+a_{3} T+a_{4} T^{2}
$$

$D V F 2$

$$
\sigma_{i v}=a_{0}+a_{1} K+a_{2} K^{2}+a_{3} T+a_{4} K T
$$

DVF 3

$$
\sigma_{i v}=a_{0}+a_{1} K+a_{2} K^{2}+a_{3} T+a_{4} T^{2}+a_{5} K T
$$

where $\sigma_{i v}=$ Black-Scholes implied volatility. $K=$ strike price. $T=$ time to maturity and $a_{0}, a_{1}, a_{2}, a_{3}, a_{4}, a_{5}$ are model parameters. In case of constant volatility assumption DVF model converges to BS $\sigma_{i v}=a_{0}$.

\subsection{The Constant-Elasticity-of-Variance (CEV) Option Pricing Model}

Utilizing and extending the framework of BS, Cox and Ross (1976) proposed the constant elasticity of variance (CEV) model. The CEV model assumes the diffusion process for the stock is

$$
d S=\mu d t+\delta S^{\beta / 2} d z
$$

and the instantaneous variance of the percentage price change or return, $\sigma^{2}$, follows deterministic relationship:

$$
\sigma^{2}(S, t)=\delta^{2} S^{(\beta-2)}
$$

If $\beta=2$, prices are lognormally distributed and the variance of returns is constant. This is the same as the well-known Black-Scholes model. If $\beta<2$, the stock price is inversely related to the volatility. When $\beta<2$, the nondividend-paying CEV call pricing formula is as follows:

$$
C=S\left[\sum_{n=0}^{\infty} g\left(S^{\prime} \mid n+1\right) G\left(K^{\prime} \mid n+1+\frac{1}{2-\beta}\right)\right]-K e^{-r \tau}\left[\sum_{n=0}^{\infty} g\left(S^{\prime} \mid n+1+\frac{1}{2-\beta}\right) G\left(K^{\prime} \mid n+1\right)\right]
$$

When $\beta>2$, the CEV call pricing formula is as follows:

$$
C=S\left[1-\sum_{n=0}^{\infty} g\left(S^{\prime} \mid n+1+\frac{1}{2-\beta}\right) G\left(K^{\prime} \mid n+1\right)\right]-K e^{-r \tau}\left[1-\sum_{n=0}^{\infty} g\left(S^{\prime} \mid n+1\right) G\left(K^{\prime} \mid n+1+\frac{1}{2-\beta}\right)\right]
$$

where

$$
\begin{aligned}
S^{\prime} & =\left[\frac{2 r e^{r \tau(2-\beta)}}{\delta^{2}(2-\beta)\left(e^{r \tau(2-\beta)}-1\right)}\right] S^{2-\beta} \\
K^{\prime} & =\left[\frac{2 r}{\delta^{2}(2-\beta)\left(e^{r \tau(2-\beta)}-1\right)}\right] K^{2-\beta}
\end{aligned}
$$


$g(x \mid m)=\frac{e^{-x} x^{m-1}}{\Gamma(m)}$ is the gamma density function

$$
G(x \mid m)=\int_{x}^{\infty} g(y \mid m) d y
$$

$C$ is the call price; $S$, the stock price; $\tau$, the time to maturity; $r$, the risk-free rate of interest; $K$, the strike price; and $\beta$ and $\delta$, the parameters of the formula. As a special case when $\beta$ of the model is equal to 2 , the model reproduces the BS. The CEV model is complex enough to incorporate the dynamics of changing volatility but at the same time simple enough to provide a closed form solution for options with only two parameters (Ang and Peterson, 1984; Lee, $\mathrm{Wu}$ and Chen, 2004).

\subsection{The Gram-Charlier Model}

The Gram-Charlier (2004) model provides a simple way to account non log-normality of asset return and accounts both excess skewness and kurtosis. Gram-Charlier model provides expansion up to the fourth order in the distribution of returns of the underlying asset. The model is derived on the conceptual framework of BS i.e. volatility is constant over time. The Gram-Charlier formula is

$$
C_{G C} \approx S_{t} \phi(d)-K e^{-R t} \phi(d-\sigma)+S_{t} \phi(d) \sigma\left[\frac{\gamma_{1 t}}{3 !} \phi^{(3)}(2 \sigma t-d)-\frac{\gamma_{2 t}}{4 !}\left(1-d^{2}+3 d \sigma t\right)-\sigma_{t}^{2}\right]
$$

Where $r$ is continuously compounded $n$-period interest rate, $\mathrm{K}$ is the strike price of the option and $\mathrm{d}$ is identical to that of BS formula. In the case if skewness and excess kurtosis are both zero, the terms inside the square brackets become zero, and the $\mathrm{GC}$ formula for the call price $\boldsymbol{C}_{\boldsymbol{G C}}$ will reduces to the BS call price.

\subsection{Hull and White Uncorrelated Stochastic Volatility Model}

Extending the constant volatility framework of BS (1973), and utilizing their year old concept of stochastic, Hull and White (1988) develop a closed form approximation for European options under new framework. However, contrary to Hull-White (1987), the 1988 version model modeled the instantaneously correlation of volatility to the asset price. They assume a square root stochastic volatility processes for a security price $\mathrm{S}$ and its return volatility:

$$
\begin{gathered}
\frac{d S}{S}=\phi d t+\sqrt{V} d Z \\
d V=\eta d t+\xi \sqrt{V} d w
\end{gathered}
$$

where $S$ is a stock price, $V$ is an instantaneous stock return variance and $d z, d w$ are Wiener processes with correlation $\rho$. $\xi$ is the instantaneous standard deviation of $d V / \sqrt{V} . \phi$ is the exponential drift rate of $S$ and $\eta(V)=a+b V$ is the instantaneous drift rate of $V$, where $a$ and $b$ are constants. Mean-reverting volatility assumes that $b$ is negative with a long-run reversion value of $-a / b$, where $a$ must be positive to maintain a positive variance.

Hull and White (1988) provide an accurate approximation from a second-order Taylor series expansion around a constant volatility specification $(\xi=0)$. Using this expansion, they derived the formula

$$
C_{H W}=C_{B S}(V)+Q_{1} \rho \xi+\left(Q_{2}+Q_{3} \rho^{2}\right) \xi^{2}+o\left(\xi^{2}\right)
$$

Where $\mathrm{C}_{\mathrm{BS}}$ is the $\mathrm{BS}$ call price and rest other is the bias term added to yield the stochastic-volatility adjusted call price

\subsection{Heston Model with Closed-form Solutions}

Heston's provided the another stochastic framework and derived a closed-form solution of a European call option on a non-dividend paying asset. His formulas is

$$
C\left(S_{t}, V_{t}, t, T\right)=S_{t} P_{1}-K e^{-r(T-t)} P_{2}
$$

where $P_{1}$, and $P_{2}$ are two probability functions, and

$$
P_{j}\left(x, V_{t}, T, K\right)=\frac{1}{2}+\frac{1}{\pi} \int_{0}^{\infty} \operatorname{Re}\left(\frac{e^{-i \phi \ln (K)} f_{j}\left(x, V_{t}, T, \phi\right)}{i \phi}\right) d \phi
$$


$x=\ln \left(S_{t}\right)$

$$
\begin{gathered}
f_{j}\left(x, V_{t}, T, \phi\right)=\exp \left\{C(T-t, \phi)+D(T-t, \phi) V_{t}+i \phi x\right\} \\
C(T-t, \phi)=r \phi i r+\frac{a}{\sigma^{2}}\left[\left(b_{j}-\rho \sigma \phi i+d\right) \tau-2 \ln \left(\frac{1-g e^{d r}}{1-g}\right)\right] \\
D(T-t, \phi)=\frac{\left(b_{j}-\rho \sigma \phi i+d\right)}{\sigma^{2}}\left(\frac{1-e^{d r}}{1-g e^{d r}}\right) \\
g=\frac{b_{j}-\rho \sigma \phi i+d}{b_{j}-\rho \sigma \phi i-d} \\
d=\sqrt{\left(\rho \sigma \phi i-b_{j}\right)^{2}-\sigma^{2}\left(2 u_{j} \phi i-\phi^{2}\right)}
\end{gathered}
$$

For $\mathrm{j}=1,2$ where,

$$
u_{1}=\frac{1}{2}, u_{21}=-\frac{1}{2}, a=\kappa \theta, b_{1}=\kappa+\lambda-\rho \sigma, b_{2}=\kappa+\lambda
$$

Theoretically such a formula looks daunting, but in reality its quite easy to evaluate. The only part that can pose a little problem in computation is the limits of the integral. As the integral cannot be evaluated exactly, but with the advancement in computational techniques it can be approximated with reasonable accuracy.

\section{Calibration of Models}

In the BS world, the implied volatility is the only parameter, which needs to be calibrated. To get an undisputable volatility parameter the best method is to infer it from the market itself which will incorporate all the available information contained in various option prices. Since all the models have one or more unknown parameters which need to be determine implicitly, for the purpose of this research we inferred all in similar way. For same we employed the non-linear least square (NLLS) loss function to imply option-related parameters from the market. The method of NLLS is a widely acceptable procedure to procure the implied parameters of the models that govern the underlying asset distribution purely from the underlying asset return and option data. The optimal set of parameters is then used to compute the models price. By using the same loss function, NLLS, we tried to provide a level playing field to all models. The idea of estimating the volatility process first and then estimating the option related parameters have been utilized here. Using this procedure, parameter estimates of all the models are obtained. The volatility and other parameters estimates obtained for day $i$ is used to value options of day $i+l$. Equation (1) exhibits the square loss objective function $f(\Omega)$

$$
f(\Omega)=\min _{\Omega} \sum_{i=1}^{n}\left[C_{o b s, i}(K, T)-C_{\text {Model }, i}\left(a_{1}, a_{2}, \ldots \ldots a_{n}\right)\right]^{2}
$$

where $\Omega$ is the set of parameter to be determined implicitly from the market data.

\section{Results}

This section reports the empirical comparison of model performances. Table 1- 4(a) explicitly states the pattern of implied volatility and out-of-sample forecasting performance of models.

\subsection{The Implied Volatility Pattern}

To determine the pricing bias between the BS model price and the market price a convenient method is to plot the BS implied volatility as a function of the exercise price. Previous studies of researchers have acknowledged the volatility smile pattern which means that the implied volatility tends to vary across exercise prices. Results of Table $1 \& 1$ (a) clearly exhibits that results are inline with the previous empirical facts, reports implied volatility is higher for OTM and ITM options but lower for ATM options, moves systematically, increases in either way from ATM. The existence of volatility smile shows the BS model systematically misprices options across moneyness-maturity groups. Incorporation of stochastic volatility in BS model might improve its performance in terms of more stability in volatility and lower price error. Thus, study of the implied volatility sets the first stage to judge the empirical performance of family of option pricing models. 


\subsection{Out-of-Sample Pricing Performance}

\section{Black-Scholes Model}

Table 2, 2(a), 3 and 3(b) exhibits that the BS model over prices medium and long term OTM and DOTM call options and under prices short term DOTM, OTM, ITM and DITM call options. Across all groups of moneyness and maturity, PME is least for ITM and DITM call options. Its evident from the tables that BS model prices ATM, ITM and DITM call options with error less than equal to 7\% in all moneyness-maturity groups. Degree of pricing bias is increases systematically and moves from short-term to long-term options for short and medium term call options. We found that the BS model prices short term ATM, ITM and DITM options more accurately with pricing error varying from $0 \%$ to $-2 \%$ only.

\section{Deterministic Volatility Functions}

We found that the DVF models under prices short term DOTM and overprices long-term DITM options. Among the three variants of DVF, DVF 1 and DVF 2 models outperforms the benchmark BS and GC model in 13 out of 15 moneyness-maturity groups. Amazingly the pricing bias of DVF 2 and DVF 3 is least in 11 out of 15 moneyness-maturity groups, outperforms all other models. For DVF models PME is generally high for DOTM call options for all maturity and low for short and medium term ITM and DITM call options. Since, incorporation of DVF 1-3 does not add any computational complexities to the BS option pricing, we suggest that this modified approach could be use as an alternative of BS.

\section{Gram-Charlier Model}

Table 2-4(a) strongly suggest that the incorporation of skewness and kurtosis into the option pricing formula yields values closer to market prices, outperformed BS in 12 out of 15 categories of moneyness-maturity . Results also justify the weakness of BS model which does not incorporate same.

\section{The Constant Elasticity of Variance Model}

Results reveals that the CEV model under prices short term OTM, ATM, ITM and DITM call options and medium term DOTM call options. The model also overprices long-term call options with percentage mean error ranging 4-9\%. The model performs better than the BS model in 9 out of 15 moneyness-maturity groups. The results of Table 2-4(a) displays that the PME of CEV model closely matches with the results of stochastic volatility models in short and medium term moneyness-maturity category, but in rest other its performance is not good. However, the pricing performance of CEV model is better than the Heston and HW model in DOTM category. The degree of mispricing of CEV model is ranging between $26 \%$ and $34 \%$ in all maturity.

\section{Hull and White Model}

HW model under prices DOTM, OTM, ITM and DITM call options with maturities less than 30 days and ITM, DITM call options of maturity less than or equal to 60 days, while over prices DOTM options for maturity group greater than 30 and 60 days. The model deeply under-prices short term DOTM and OTM options, error varies from $27 \%$ and $6 \%$. HW model prices short and medium term ATM, ITM and DITM options more accurately with $0-3 \%$ degree of price bias. For all moneyness-maturity groups, HW model generally produces prices that are very close to BS prices, this may be due to fact that HW model is merely an stochastic extensions of BS model. MAPE of HW model is higher in case of ATM options for short maturity options as compared to CEV and Hestion while lower for short-term DOTM options compared to Heston.

\section{Heston Model}

Table 1 reveals that the implied volatility of the Heston model matches the market-implied volatility better than the BS, GC CEV and HW models. However Table 1(a) evident that implied volatility computed from the Heston model price is always higher than the implied volatility computed from the market price for in-the-money but lower for out-of-the-money options. The out-of-sample forecast ability of the Heston model is superior to classical BS model in 12 out of 15 moneyness-maturity groups (PME is lower in 12 whereas MAPE is lower in 13 out of 15 moneyness-maturity groups). Our results strongly support the view that addition of volatility as a random variant improves the pricing bias significantly, but inclusion of higher numbers of unknown parameters makes the computational process complex and even after that the pricing bias is not eliminating completely. Despite the computational complexity, the Heston Model is the most widely used stochastic volatility (SV) models today. Its attractiveness lies in the powerful duality of its tractability and robustness relative to other models.

After cross-comparison, we found that none of the models dominates the others. In other words, no model reproduces market prices that give relative errors lower than the other models for all strikes and maturities. Thus, 
our key objective, which was to found the best model, not achieved. Therefore we shifted our focus on the identification of finding the best alternative among the family of models producing lowest error across moneyness and maturity. The DVF, CEV and the Heston model appear to be pricing better than the rest others in the short maturity ITM category. For short maturity and ATM category, the error of HW is lowest. However, the classical BS model also comes in handy at least for ATM, ITM and DITM options. For medium and long maturity results are not as diverse as for short maturity options. Although, performance of all the models are closely competitive but pricing performance of DVF and Heston model appears to be better in peer group as their relative errors are much lower. After rigorous churning of specific data taken across various models we deduced that the best model to be used for option pricing is the deterministic DVF and stochastic Heston model as they outperformed and surpassed other models in most of moneyness-maturities.

\subsection{Choosing between Models}

Since all the models found to be produce relative errors with respect to the market in all option categories, therefore we conclude that there is no model that can fully approximate the market. The possible reasons are either estimated parameter values are incorrect or models are not capable of capturing the variations of the option market. Both the possibility is highly likely in this research. The second possibility is more likely as in reality option prices are determined by non-quantifiable factors such as demand and supply, than just the quantifiable underlying assets. Table 5 lists the models categorically best for option pricing.

\section{Conclusion}

Our result shows that the DVF and Heston model improves pricing error significantly compared to others model. From the results of the simulation, we have found that, for Nifty index options: None of the models can fully reproduce the market prices since all of them produce relative errors with respect to the market. Judged on internal parameter consistency, all models are mis-specified, with the DVF and Heston model the least and the BS the most misspecified. There is no model that dominates the others by producing prices that are in closer agreement with the market prices in all option categories (moneyness-maturity category). Out-of-sample (OTM) pricing errors are the highest for the Black-Scholes and Hull white Stochastic Volatility model, the second highest for the Gram-Charlier, and the lowest for the Heston Stochastic model. Out of all the models, Practitioner Black-Scholes, Constant Elasticity of Variance, and Heston model is one of the best for pricing short and medium term in-the-money and deep-in-the-money Nifty index options. Overall, the stochastic volatility model typically reduces the BS pricing errors by $20 \%$ to $30 \%$. Stochastic Volatility models do not significantly improve the performance over deterministic volatility models of the BS, DVF, CEV, Gram-Charlier models. The Heston model, comparing to other models, is good in for both short and long maturity, deep-out-of-the-money and short maturity, in-the-money and deep-in-the-money option categories. In the realm of financial option pricing the model is most popular and trustworthy because of its simplicity and analytical tractability and thus used globally to fix the base price of options traded on the bourses of almost all the option exchanges.

\section{References}

Abken, P. A., \& Nandi, S. (1996). Options and Volatility. Economic Review, 81(3), 21-35.

Ang, J. S., \& Peterson, D. R. (1984). Empirical Properties of the Elasticity Coefficient in the Constant Elasticity of Variance Model. Finance Review, 19(4), 372-380. http://dx.doi.org/10.1111/j.1540-6288.1984.tb00657.x

Bachelier, L. (1900). Th'eorie de la Sp'eculation. Annales Scientifiques de l'École Normale Supérieure, 17, 21-86. English translation in P. Cootner (ed.), The Random Character of Stock Prices. MIT Press, 1964, reprinted Risk Books, London 2000.

Backus, D., Foresi, S., \& Wu, L. (1997). Accounting for Biases in Black- Scholes. CRIF Working Paper Series, Paper 30. New York University.

Ball, C. A., \& Roma, A. (1994). Stochastic Volatility Option Pricing. Journal of Financial and Quantitative Analysis, 29(4), 589-607. http://dx.doi.org/10.2307/2331111

Black, F., \& Scholes, M. (1973). The Pricing of Options and Corporate Liabilities. Journal of Political Economy, 81(3), 637-659. http://dx.doi.org/10.1086/260062

Brockman, P., \& Chowdhury, M. (1997). Deterministic versus Stochastic Volatilities: Implications for Option Pricing Models. Applied Financial Economics, 7(5), 499-505. http://dx.doi.org/10.1080/096031097333367

Christoffersen, P., \& Jacobs, K. (2004). The Importance of the Loss Function in Option Pricing. Journal of Financial Econometrics, 72(2), 291-318. http://dx.doi.org/10.1016/j.jfineco.2003.02.001 
Coleman, T. F., \& Li, Y. (1996). An interior, trust region approach for nonlinear minimization subject to bounds. SIAM Journal on Optimization, 6, 418-445. http://dx.doi.org/10.1137/0806023

Cont, R. (2001). Empirical properties of asset returns: stylized facts and statistical issues. Quantitative Finance, 1(2), 223-236. http://dx.doi.org/10.1080/713665670

Cox, J., \& Ross, S. (1976). The Valuation of Options for Alternative Stochastic Processes. Journal of Financial Economics, 3(1-2), 145-166. http://dx.doi.org/10.1016/0304-405X(76)90023-4

Dumas, B., Fleming, J., \& Whaley, R. (1998). Implementing Volatility Functions: Empirical Tests. Journal of Finance, 53(6), 2059-2106. http://dx.doi.org/10.1111/0022-1082.00083

Heston, S. (1993). A Closed-Form Solution for Options with Stochastic Volatility, with Applications to Bond and Currency Options. Review of Financial Studies, 6(2), 327-343. http://dx.doi.org/10.1093/rfs/6.2.327

Heston, S., \& Nandi, S. (2000). A Closed-Form GARCH Option Valuation Model. Review of Financial Studies, 13(3), 585-625. http://dx.doi.org/10.1093/rfs/13.3.585

Heynen, R. (1993). An Empirical Investigation of Observed Smile Patterns. Review of Future Markets, 13(2), 317-353.

Hull, J., \& White, A. (1987). The Pricing of Options on Assets with Stochastic Volatility. Journal of Finance, 42(2), 281-300. http://dx.doi.org/10.1111/j.1540-6261.1987.tb02568.x

Hull, J. C., \& White, A. (1988). An analysis of the bias in option pricing caused by a stochastic volatility. Advances in Futures and Options Research, 3, 27-61

Lee, C. F., T. Wu, \& Chen, R. (2004). The Constant Elasticity of Variance Models: New Evidence from S\&P 500 Index Options. Review of Pacific Basin Financial Markets and Policies, 7(2), 173-190. http://dx.doi.org/10.1142/S021909150400010X

Rubinstein, M. (1985). Non-Parametric Tests of Alternative Option Pricing Models Using all Reported Trades and Quotes on the 30 Most Active CBOE Option Classes from August 23, 1976 Trough August 31, 1978. Journal of Finance, 40(2), 455-480. http://dx.doi.org/10.1111/j.1540-6261.1985.tb04967.x

Rubinstein, M. (1994). Implied Binomial Trees, Journal of Finance, 49(3), 771-818. http://dx.doi.org/10.1111/j.1540-6261.1994.tb00079.x

Scott, L. O. (1987). Option Pricing when the Variance Changes Randomly: Theory, Estimation and an Application. The journal of Financial and Quantitative Analysis, 22(4), 419-438. http://dx.doi.org/10.2307/2330793

Stein, E. M., \& Stein, J. C. (1991). Stock Price Distributions with Stochastic Volatility: An Analytic Approach. Review of Financial Studies, 4(4), 727-752. http://dx.doi.org/10.1093/rfs/4.4.727

Wiggins, J. B. (1987). Option Values under Stochastic Volatilities. Theory and Empirical Estimates. Journal of Financial Economics, 19(2), 351-372. http://dx.doi.org/10.1016/0304-405X(87)90009-2 


\section{Appendix}

Table 1. Black-Scholes and Alternate Option Pricing Models average implied volatility moneyness-maturity bias

\begin{tabular}{|c|c|c|c|c|c|c|c|c|c|c|c|c|c|c|c|c|c|c|}
\hline \multirow{3}{*}{ Models } & \multicolumn{18}{|c|}{ Implied Volatility } \\
\hline & \multicolumn{6}{|c|}{ Time to maturity $(\mathrm{T} \leq 30)$} & \multicolumn{6}{|c|}{ Time to maturity $\quad(30<\mathrm{T} \leq 60)$} & \multicolumn{6}{|c|}{ Time to maturity $\quad(T>60)$} \\
\hline & DOTM & OTM & ATM & ITM & DITM & All & DOTM & OTM & ATM & ITM & DITM & All & DOTM & OTM & ATM & ITM & DITM & All \\
\hline \multirow{2}{*}{ BS } & 0.39 & 0.37 & 0.35 & 0.36 & 0.37 & 0.36 & 0.39 & 0.37 & 0.35 & 0.37 & 0.39 & 0.37 & 0.40 & 0.36 & 0.33 & 0.38 & 0.42 & 0.35 \\
\hline & 0.12 & 0.12 & 0.11 & 0.12 & 0.13 & 0.12 & 0.12 & 0.12 & 0.12 & 0.12 & 0.14 & 0.12 & 0.13 & 0.11 & 0.09 & 0.10 & 0.11 & 0.11 \\
\hline \multirow{2}{*}{ DVF 1} & 0.43 & 0.40 & 0.38 & 0.39 & 0.41 & 0.40 & 0.43 & 0.39 & 0.37 & 0.39 & 0.43 & 0.39 & 0.43 & 0.39 & 0.35 & 0.42 & 0.49 & 0.38 \\
\hline & 0.14 & 0.14 & 0.13 & 0.14 & 0.14 & 0.14 & 0.15 & 0.15 & 0.14 & 0.15 & 0.17 & 0.15 & 0.15 & 0.13 & 0.12 & 0.14 & 0.16 & 0.13 \\
\hline \multirow{2}{*}{ DVF 2} & 0.42 & 0.39 & 0.38 & 0.39 & 0.40 & 0.39 & 0.42 & 0.39 & 0.37 & 0.39 & 0.43 & 0.39 & 0.42 & 0.38 & 0.35 & 0.41 & 0.49 & 0.37 \\
\hline & 0.15 & 0.14 & 0.14 & 0.15 & 0.15 & 0.15 & 0.15 & 0.15 & 0.14 & 0.15 & 0.17 & 0.15 & 0.15 & 0.13 & 0.12 & 0.14 & 0.16 & 0.13 \\
\hline \multirow{2}{*}{ DVF 3} & 0.42 & 0.40 & 0.38 & 0.39 & 0.41 & 0.39 & 0.43 & 0.40 & 0.38 & 0.39 & 0.43 & 0.39 & 0.43 & 0.39 & 0.35 & 0.41 & 0.49 & 0.38 \\
\hline & 0.15 & 0.14 & 0.14 & 0.15 & 0.15 & 0.14 & 0.15 & 0.15 & 0.15 & 0.15 & 0.18 & 0.15 & 0.15 & 0.13 & 0.12 & 0.15 & 0.17 & 0.14 \\
\hline \multirow{2}{*}{ GC } & 0.40 & 0.37 & 0.36 & 0.37 & 0.38 & 0.37 & 0.40 & 0.37 & 0.35 & 0.37 & 0.41 & 0.37 & 0.39 & 0.36 & 0.32 & 0.37 & 0.40 & 0.35 \\
\hline & 0.26 & 0.21 & 0.21 & 0.25 & 0.25 & 0.23 & 0.24 & 0.17 & 0.18 & 0.12 & 0.32 & 0.19 & 0.13 & 0.11 & 0.09 & 0.09 & 0.10 & 0.11 \\
\hline \multirow{2}{*}{ CEV } & 0.32 & 0.30 & 0.28 & 0.29 & 0.30 & 0.30 & 0.32 & 0.30 & 0.28 & 0.30 & 0.32 & 0.30 & 0.33 & 0.29 & 0.26 & 0.31 & 0.34 & 0.29 \\
\hline & 0.11 & 0.10 & 0.10 & 0.10 & 0.11 & 0.10 & 0.11 & 0.11 & 0.10 & 0.11 & 0.12 & 0.11 & 0.11 & 0.10 & 0.08 & 0.09 & 0.10 & 0.10 \\
\hline \multirow{2}{*}{ HW } & 0.39 & 0.37 & 0.35 & 0.36 & 0.37 & 0.36 & 0.39 & 0.37 & 0.35 & 0.37 & 0.39 & 0.37 & 0.40 & 0.36 & 0.33 & 0.38 & 0.42 & 0.35 \\
\hline & 0.12 & 0.12 & 0.11 & 0.12 & 0.13 & 0.12 & 0.12 & 0.12 & 0.12 & 0.12 & 0.13 & 0.12 & 0.13 & 0.11 & 0.09 & 0.10 & 0.11 & 0.11 \\
\hline \multirow{2}{*}{ Heston } & 0.45 & 0.42 & 0.40 & 0.41 & 0.43 & 0.42 & 0.45 & 0.41 & 0.39 & 0.42 & 0.45 & 0.42 & 0.47 & 0.41 & 0.37 & 0.46 & 0.52 & 0.40 \\
\hline & 0.17 & 0.16 & 0.15 & 0.16 & 0.17 & 0.16 & 0.17 & 0.16 & 0.15 & 0.15 & 0.19 & 0.16 & 0.17 & 0.15 & 0.13 & 0.16 & 0.17 & 0.15 \\
\hline $\begin{array}{c}\text { No. of } \\
\text { Observations }\end{array}$ & 772 & 938 & 1697 & 414 & 222 & 4043 & 491 & 632 & 1085 & 233 & 120 & 2561 & 153 & 235 & 432 & 22 & 9 & 851 \\
\hline
\end{tabular}

Table 1(a). Black-Scholes and Alternate Option Pricing Models average implied volatility moneyness bias

\begin{tabular}{|c|c|c|c|c|c|c|c|}
\hline \multirow{2}{*}{ Models } & & \multicolumn{5}{|c|}{ Moneyness $(\mathrm{x}=\mathrm{S} / \mathrm{K}-1)$} & \multirow[b]{2}{*}{ Overall } \\
\hline & & DOTM & OTM & ATM & ITM & DITM & \\
\hline \multirow[t]{2}{*}{ BS } & Average & 0.39 & 0.36 & 0.35 & 0.36 & 0.38 & 0.36 \\
\hline & Std Dev & 0.12 & 0.12 & 0.11 & 0.12 & 0.13 & 0.12 \\
\hline \multirow[t]{2}{*}{ DVF 1} & Average & 0.43 & 0.39 & 0.37 & 0.39 & 0.42 & 0.39 \\
\hline & Std Dev & 0.14 & 0.14 & 0.14 & 0.14 & 0.15 & 0.14 \\
\hline \multirow[t]{2}{*}{ DVF 2} & Average & 0.42 & 0.39 & 0.37 & 0.39 & 0.41 & 0.39 \\
\hline & Std Dev & 0.15 & 0.14 & 0.14 & 0.15 & 0.16 & 0.15 \\
\hline \multirow[t]{2}{*}{ DVF 3} & Average & 0.43 & 0.39 & 0.37 & 0.39 & 0.41 & 0.39 \\
\hline & Std Dev & 0.15 & 0.14 & 0.14 & 0.15 & 0.16 & 0.14 \\
\hline \multirow[t]{2}{*}{$\mathrm{GC}$} & Average & 0.40 & 0.37 & 0.35 & 0.37 & 0.39 & 0.37 \\
\hline & Std Dev & 0.24 & 0.19 & 0.19 & 0.21 & 0.27 & 0.21 \\
\hline \multirow[t]{2}{*}{ CEV } & Average & 0.32 & 0.30 & 0.28 & 0.30 & 0.31 & 0.30 \\
\hline & Std Dev & 0.11 & 0.10 & 0.10 & 0.10 & 0.11 & 0.10 \\
\hline \multirow[t]{2}{*}{ HW } & Average & 0.39 & 0.36 & 0.35 & 0.36 & 0.38 & 0.36 \\
\hline & Std Dev & 0.12 & 0.12 & 0.11 & 0.12 & 0.13 & 0.12 \\
\hline \multirow[t]{2}{*}{ Heston } & Average & 0.45 & 0.41 & 0.39 & 0.42 & 0.44 & 0.41 \\
\hline & Std Dev & 0.17 & 0.16 & 0.15 & 0.16 & 0.18 & 0.16 \\
\hline \multicolumn{2}{|c|}{ Total } & 1416 & 1805 & 3214 & 669 & 351 & 7455 \\
\hline
\end{tabular}


Table 2. Black-Scholes and Alternate Option Pricing Models out of sample Percentage Mean Error (PME) moneyness-maturity bias

\begin{tabular}{|c|c|c|c|c|c|c|c|c|c|c|c|c|c|c|c|c|c|c|}
\hline \multirow{3}{*}{ Models } & \multicolumn{18}{|c|}{ PME } \\
\hline & \multicolumn{6}{|c|}{ Time to maturity $\quad(\mathrm{T} \leq 30)$} & \multicolumn{6}{|c|}{ Time to maturity $\quad(30<\mathrm{T} \leq 60)$} & \multicolumn{6}{|c|}{ Time to maturity $\quad(\mathrm{T}>60)$} \\
\hline & DOTM & OTM & ATM & ITM & DITM & All & DOTM & OTM & ATM & ITM & DITM & All & DOTM & OTM & ATM & ITM & DITM & All \\
\hline \multirow{2}{*}{ BS } & -0.27 & -0.07 & -0.03 & -0.04 & -0.03 & -0.09 & 0.10 & 0.10 & 0.00 & -0.01 & -0.02 & 0.04 & 0.15 & 0.11 & 0.07 & 0.00 & 0.04 & 0.09 \\
\hline & 0.53 & 0.42 & 0.17 & 0.05 & 0.04 & 0.34 & 0.34 & 0.35 & 0.11 & 0.07 & 0.05 & 0.25 & 0.44 & 0.24 & 0.50 & 0.10 & 0.12 & 0.42 \\
\hline \multirow{2}{*}{ DVF 1} & -0.06 & 0.02 & 0.02 & 0.00 & 0.00 & 0.00 & 0.01 & 0.03 & 0.00 & 0.02 & 0.02 & 0.01 & 0.02 & 0.05 & 0.04 & 0.01 & 0.06 & 0.04 \\
\hline & 1.25 & 0.48 & 0.17 & 0.06 & 0.05 & 0.61 & 0.37 & 0.30 & 0.11 & 0.07 & 0.06 & 0.23 & 0.47 & 0.31 & 0.51 & 0.12 & 0.13 & 0.44 \\
\hline \multirow{2}{*}{ DVF 2} & -0.16 & -0.05 & 0.01 & 0.00 & 0.00 & -0.04 & 0.01 & 0.03 & 0.01 & 0.01 & 0.02 & 0.01 & 0.00 & 0.01 & 0.03 & 0.00 & 0.04 & 0.02 \\
\hline & 1.09 & 0.53 & 0.15 & 0.06 & 0.05 & 0.55 & 0.36 & 0.30 & 0.11 & 0.08 & 0.06 & 0.23 & 0.41 & 0.22 & 0.51 & 0.10 & 0.10 & 0.42 \\
\hline \multirow{2}{*}{ DVF 3} & -0.08 & 0.00 & 0.03 & 0.01 & 0.00 & 0.00 & 0.00 & 0.01 & 0.00 & 0.01 & 0.01 & 0.00 & -0.02 & 0.03 & 0.04 & -0.01 & 0.05 & 0.03 \\
\hline & 1.11 & 0.52 & 0.16 & 0.07 & 0.05 & 0.56 & 0.38 & 0.29 & 0.11 & 0.07 & 0.06 & 0.24 & 0.44 & 0.26 & 0.53 & 0.09 & 0.10 & 0.44 \\
\hline \multirow{2}{*}{ GC } & -0.62 & -0.28 & -0.02 & -0.02 & -0.02 & -0.20 & -0.06 & 0.04 & 0.00 & 0.00 & 0.00 & 0.00 & 0.07 & 0.08 & 0.07 & 0.01 & 0.03 & 0.07 \\
\hline & 0.64 & 0.46 & 0.17 & 0.07 & 0.06 & 0.44 & 0.34 & 0.33 & 0.11 & 0.07 & 0.05 & 0.24 & 0.41 & 0.23 & 0.50 & 0.09 & 0.09 & 0.42 \\
\hline \multirow{2}{*}{ CEV } & -0.47 & -0.21 & -0.05 & -0.02 & -0.01 & -0.16 & -0.08 & 0.01 & 0.00 & 0.02 & 0.02 & -0.01 & 0.04 & 0.06 & 0.09 & 0.04 & 0.08 & 0.07 \\
\hline & 0.39 & 0.34 & 0.15 & 0.06 & 0.04 & 0.30 & 0.26 & 0.30 & 0.10 & 0.07 & 0.05 & 0.20 & 0.38 & 0.21 & 0.48 & 0.09 & 0.12 & 0.39 \\
\hline \multirow{2}{*}{ HW } & -0.27 & -0.06 & -0.03 & -0.04 & -0.03 & -0.08 & 0.10 & 0.10 & 0.00 & -0.01 & -0.02 & 0.04 & 0.15 & 0.11 & 0.07 & 0.00 & 0.04 & 0.10 \\
\hline & 0.53 & 0.42 & 0.17 & 0.05 & 0.04 & 0.34 & 0.34 & 0.35 & 0.11 & 0.07 & 0.05 & 0.25 & 0.44 & 0.24 & 0.50 & 0.10 & 0.12 & 0.42 \\
\hline \multirow{2}{*}{ Heston } & -0.01 & 0.07 & 0.03 & -0.01 & -0.02 & 0.02 & 0.08 & 0.06 & 0.00 & 0.00 & -0.01 & 0.03 & 0.02 & 0.00 & 0.03 & -0.01 & 0.02 & 0.02 \\
\hline & 1.03 & 0.46 & 0.18 & 0.06 & 0.04 & 0.51 & 0.33 & 0.31 & 0.11 & 0.07 & 0.05 & 0.23 & 0.36 & 0.21 & 0.50 & 0.09 & 0.11 & 0.40 \\
\hline $\begin{array}{c}\text { No. of } \\
\text { Observations }\end{array}$ & 772 & 938 & 1697 & 414 & 222 & 4043 & 491 & 632 & 1085 & 233 & 120 & 2561 & 153 & 235 & 432 & 22 & 9 & 851 \\
\hline
\end{tabular}

Table 2(a). Black-Scholes and Alternate Option Pricing Models out of sample Percentage Mean Error (PME) moneyness bias

\begin{tabular}{|c|c|c|c|c|c|c|c|}
\hline \multirow{2}{*}{ Models } & & \multicolumn{5}{|c|}{ Moneyness $(\mathrm{x}=\mathrm{S} / \mathrm{K}-1)$} & \multirow[b]{2}{*}{ Overall } \\
\hline & & DOTM & OTM & ATM & ITM & DITM & \\
\hline \multirow[t]{2}{*}{$\mathrm{BS}$} & Average & -0.10 & 0.02 & -0.01 & -0.03 & -0.02 & -0.02 \\
\hline & Std Dev & 0.50 & 0.39 & 0.23 & 0.06 & 0.05 & 0.33 \\
\hline \multirow[t]{2}{*}{ DVF 1} & Average & -0.03 & 0.02 & 0.02 & 0.01 & 0.00 & 0.01 \\
\hline & Std Dev & 0.96 & 0.41 & 0.23 & 0.07 & 0.06 & 0.49 \\
\hline \multirow[t]{2}{*}{ DVF 2} & Average & -0.08 & -0.02 & 0.01 & 0.01 & 0.00 & -0.01 \\
\hline & Std Dev & 0.85 & 0.43 & 0.23 & 0.07 & 0.06 & 0.45 \\
\hline \multirow[t]{2}{*}{ DVF 3} & Average & -0.05 & 0.01 & 0.02 & 0.01 & 0.00 & 0.00 \\
\hline & Std Dev & 0.86 & 0.43 & 0.24 & 0.07 & 0.06 & 0.46 \\
\hline \multirow[t]{2}{*}{ GC } & Average & -0.35 & -0.12 & 0.00 & -0.01 & -0.01 & -0.10 \\
\hline & Std Dev & 0.61 & 0.43 & 0.23 & 0.07 & 0.06 & 0.39 \\
\hline \multirow[t]{2}{*}{$\mathrm{CEV}$} & Average & -0.28 & -0.10 & -0.01 & 0.00 & 0.00 & -0.08 \\
\hline & Std Dev & 0.41 & 0.33 & 0.22 & 0.06 & 0.05 & 0.30 \\
\hline \multirow[t]{2}{*}{ HW } & Average & -0.09 & 0.02 & -0.01 & -0.03 & -0.02 & -0.02 \\
\hline & Std Dev & 0.50 & 0.39 & 0.23 & 0.06 & 0.05 & 0.33 \\
\hline \multirow[t]{2}{*}{ Heston } & Average & 0.02 & 0.05 & 0.02 & -0.01 & -0.01 & 0.03 \\
\hline & Std Dev & 0.79 & 0.39 & 0.23 & 0.06 & 0.05 & 0.42 \\
\hline \multicolumn{2}{|c|}{ Total } & 1416 & 1805 & 3214 & 669 & 351 & 7455 \\
\hline
\end{tabular}


Table 3. Black-Scholes and Alternate Option Pricing Models Mean Absolute Pricing Error (MAPE) moneyness-maturity bias

\begin{tabular}{|c|c|c|c|c|c|c|c|c|c|c|c|c|c|c|c|c|c|c|}
\hline \multirow{3}{*}{ Models } & \multicolumn{18}{|c|}{ MAPE } \\
\hline & \multicolumn{6}{|c|}{ Time to maturity $\quad(\mathrm{T} \leq 30)$} & \multicolumn{6}{|c|}{ Time to maturity $\quad(30<\mathrm{T} \leq 60)$} & \multicolumn{6}{|c|}{ Time to maturity $\quad(\mathrm{T}>60)$} \\
\hline & DOTM & OTM & ATM & ITM & DITM & All & DOTM & OTM & ATM & ITM & DITM & All & DOTM & OTM & ATM & ITM & DITM & All \\
\hline \multirow{2}{*}{ BS } & 6.36 & 8.75 & 14.24 & 17.25 & 17.75 & 11.96 & 13.58 & 15.98 & 16.78 & 20.76 & 19.71 & 16.47 & 26.26 & 22.51 & 26.45 & 34.26 & 43.22 & 25.71 \\
\hline & 10.34 & 10.13 & 15.86 & 19.65 & 21.31 & 15.14 & 17.10 & 19.13 & 21.80 & 20.23 & 24.37 & 20.40 & 30.51 & 23.06 & 31.74 & 26.85 & 44.19 & 29.47 \\
\hline \multirow{2}{*}{ DVF 1} & 6.49 & 8.79 & 12.89 & 15.47 & 16.49 & 11.18 & 12.60 & 13.76 & 16.41 & 20.63 & 22.27 & 15.68 & 23.60 & 25.91 & 26.71 & 40.11 & 40.03 & 26.42 \\
\hline & 10.81 & 10.85 & 15.40 & 18.73 & 21.71 & 14.86 & 17.06 & 16.64 & 22.43 & 20.53 & 27.42 & 20.43 & 29.04 & 31.67 & 29.40 & 30.74 & 54.12 & 30.41 \\
\hline \multirow{2}{*}{ DVF 2} & 6.88 & 8.03 & 11.97 & 15.29 & 15.91 & 10.64 & 11.95 & 13.03 & 16.69 & 22.11 & 22.25 & 15.63 & 19.50 & 20.65 & 26.08 & 35.23 & 28.11 & 23.66 \\
\hline & 10.99 & 9.73 & 14.37 & 19.32 & 21.88 & 14.31 & 16.91 & 16.64 & 24.47 & 26.05 & 28.01 & 22.03 & 22.35 & 20.58 & 31.04 & 25.54 & 43.25 & 27.21 \\
\hline \multirow{2}{*}{ DVF 3} & 6.73 & 8.82 & 13.29 & 15.91 & 16.04 & 11.42 & 12.70 & 13.73 & 16.71 & 21.03 & 21.91 & 15.84 & 21.84 & 22.85 & 27.94 & 30.98 & 29.02 & 25.53 \\
\hline & 10.69 & 9.93 & 15.43 & 20.25 & 22.49 & 14.99 & 18.58 & 17.45 & 22.25 & 22.09 & 27.55 & 20.92 & 24.79 & 25.71 & 33.04 & 22.39 & 42.33 & 29.72 \\
\hline \multirow{2}{*}{ GC } & 9.13 & 11.47 & 13.77 & 18.20 & 18.95 & 13.09 & 13.39 & 14.96 & 16.64 & 20.09 & 19.54 & 16.05 & 22.38 & 21.25 & 27.00 & 33.16 & 31.95 & 24.80 \\
\hline & 11.83 & 11.31 & 16.15 & 22.11 & 24.29 & 16.01 & 16.62 & 19.27 & 21.83 & 20.26 & 25.43 & 20.43 & 24.63 & 22.36 & 31.32 & 22.05 & 31.41 & 27.82 \\
\hline \multirow{2}{*}{ CEV } & 6.19 & 8.36 & 13.48 & 14.74 & 13.89 & 11.05 & 10.54 & 12.64 & 15.72 & 19.86 & 19.99 & 14.54 & 20.06 & 19.95 & 27.02 & 31.85 & 54.18 & 24.23 \\
\hline & 9.96 & 9.25 & 14.97 & 17.97 & 19.17 & 14.01 & 12.22 & 15.59 & 20.31 & 20.99 & 22.81 & 18.33 & 23.33 & 20.08 & 30.84 & 27.05 & 39.30 & 27.32 \\
\hline \multirow{2}{*}{ HW } & 6.29 & 8.73 & 14.28 & 17.25 & 17.76 & 11.96 & 13.52 & 16.03 & 16.85 & 20.77 & 19.69 & 16.50 & 26.02 & 22.51 & 26.20 & 34.26 & 43.22 & 25.54 \\
\hline & 10.32 & 10.14 & 15.85 & 19.66 & 21.31 & 15.15 & 17.11 & 19.12 & 21.84 & 20.23 & 24.38 & 20.42 & 30.51 & 23.06 & 31.75 & 26.85 & 44.19 & 29.46 \\
\hline \multirow{2}{*}{ Heston } & 7.15 & 9.00 & 12.86 & 14.35 & 15.17 & 11.15 & 13.00 & 14.65 & 16.25 & 19.48 & 19.01 & 15.66 & 20.92 & 20.24 & 24.52 & 33.45 & 34.02 & 23.02 \\
\hline & 11.40 & 10.64 & 15.25 & 18.14 & 19.67 & 14.51 & 16.55 & 18.64 & 21.44 & 20.33 & 24.74 & 20.06 & 23.97 & 20.12 & 30.38 & 21.77 & 36.84 & 26.73 \\
\hline $\begin{array}{c}\text { No. of } \\
\text { Observations }\end{array}$ & 772 & 938 & 1697 & 414 & 222 & 4043 & 491 & 632 & 1085 & 233 & 120 & 2561 & 153 & 235 & 432 & 22 & 9 & 851 \\
\hline
\end{tabular}

Table 3(a). Black-Scholes and Alternate Option Pricing Models Mean Absolute Pricing Error (MAPE) moneyness bias

\begin{tabular}{|c|c|c|c|c|c|c|c|}
\hline \multirow{2}{*}{ Models } & & \multicolumn{5}{|c|}{ Moneyness $(\mathrm{x}=\mathrm{S} / \mathrm{K}-1)$} & \multirow[b]{2}{*}{ Overall } \\
\hline & & DOTM & OTM & ATM & ITM & DITM & \\
\hline \multirow[t]{2}{*}{ BS } & Average & 11.01 & 13.07 & 16.74 & 19.03 & 19.07 & 15.08 \\
\hline & Std Dev & 17.28 & 16.57 & 21.08 & 20.35 & 23.44 & 19.62 \\
\hline \multirow[t]{2}{*}{ DVF 1} & Average & 10.46 & 12.76 & 15.93 & 18.08 & 19.07 & 14.47 \\
\hline & Std Dev & 16.85 & 17.87 & 20.77 & 20.39 & 25.28 & 19.78 \\
\hline \multirow[t]{2}{*}{ DVF 2} & Average & 10.00 & 11.43 & 15.46 & 18.32 & 18.39 & 13.84 \\
\hline & Std Dev & 15.32 & 14.80 & 21.50 & 22.52 & 24.97 & 19.49 \\
\hline \multirow[t]{2}{*}{ DVF 3} & Average & 10.43 & 12.37 & 16.41 & 18.19 & 18.38 & 14.55 \\
\hline & Std Dev & 16.46 & 16.28 & 21.50 & 21.22 & 25.08 & 19.81 \\
\hline \multirow[t]{2}{*}{ GC } & Average & 12.04 & 13.96 & 16.52 & 19.35 & 19.49 & 15.44 \\
\hline & Std Dev & 15.93 & 16.48 & 21.18 & 21.62 & 24.88 & 19.59 \\
\hline \multirow[t]{2}{*}{ CEV } & Average & 9.19 & 11.37 & 16.05 & 17.09 & 17.01 & 13.75 \\
\hline & Std Dev & 13.51 & 14.02 & 20.12 & 19.72 & 22.11 & 17.99 \\
\hline \multirow[t]{2}{*}{ HW } & Average & 10.93 & 13.08 & 16.75 & 19.04 & 19.07 & 15.07 \\
\hline & Std Dev & 17.26 & 16.57 & 21.07 & 20.35 & 23.44 & 19.62 \\
\hline \multirow[t]{2}{*}{ Heston } & Average & 10.67 & 12.44 & 15.57 & 16.76 & 16.97 & 14.05 \\
\hline & Std Dev & 15.72 & 15.77 & 20.40 & 19.42 & 22.24 & 18.67 \\
\hline \multicolumn{2}{|c|}{ Total } & 1416 & 1805 & 3214 & 669 & 351 & 7455 \\
\hline
\end{tabular}


Table 4. Moneyness-Maturity Statistics of S\&P CNX Nifty 50 Index Call Options (prices in INR) for the period of Jan 1, 2008 to December 31, 2008 (Moneyness is defined as $x=S / K-1$, where $S$ denotes the closing value of the S\&P CNX Nifty 50 index and $K$ denotes the exercise price of the option)

\begin{tabular}{|c|c|c|c|c|c|c|c|c|c|c|c|c|c|c|c|c|c|c|}
\hline \multirow{3}{*}{ Models } & \multicolumn{18}{|c|}{ MAPE } \\
\hline & \multicolumn{6}{|c|}{ Time to maturity $(\mathrm{T} \leq 30)$} & \multicolumn{6}{|c|}{ Time to maturity $\quad(30<\mathrm{T} \leq 60)$} & \multicolumn{6}{|c|}{ Time to maturity $\quad(T>60)$} \\
\hline & DOTM & OTM & ATM & ITM & DITM & All & DOTM & OTM & ATM & ITM & DITM & All & DOTM & OTM & ATM & ITM & DITM & All \\
\hline \multirow{2}{*}{ BS } & 14.06 & 35.12 & 138.50 & 347.89 & 498.72 & 131.97 & 59.69 & 99.83 & 219.86 & 399.59 & 525.45 & 190.20 & 111.74 & 150.96 & 265.86 & 442.87 & 542.40 & 213.92 \\
\hline & 19.32 & 31.23 & 74.95 & 82.31 & 115.37 & 145.21 & 43.36 & 51.20 & 80.10 & 72.91 & 106.15 & 140.15 & 66.46 & 50.36 & 73.67 & 73.07 & 53.55 & 106.04 \\
\hline \multirow{2}{*}{ DVF 1} & 15.87 & 38.82 & 147.30 & 360.35 & 510.54 & 138.80 & 53.49 & 93.97 & 221.04 & 409.80 & 541.72 & 189.76 & 97.90 & 142.42 & 259.49 & 444.37 & 551.82 & 205.98 \\
\hline & 19.56 & 34.35 & 79.44 & 84.63 & 114.58 & 149.32 & 37.55 & 48.80 & 82.58 & 73.86 & 105.44 & 145.64 & 56.20 & 56.92 & 79.92 & 77.17 & 46.00 & 110.79 \\
\hline \multirow{2}{*}{ DVF 2} & 14.32 & 36.69 & 146.54 & 361.60 & 510.94 & 137.84 & 54.96 & 94.87 & 222.12 & 409.04 & 541.34 & 190.63 & 95.22 & 137.69 & 257.23 & 442.42 & 545.38 & 202.93 \\
\hline & 17.69 & 32.19 & 78.96 & 84.52 & 113.98 & 149.83 & 40.64 & 49.78 & 82.85 & 76.38 & 106.15 & 145.63 & 47.83 & 46.58 & 79.50 & 79.24 & 34.31 & 108.94 \\
\hline \multirow{2}{*}{ DVF 3} & 14.98 & 38.27 & 148.04 & 362.15 & 511.59 & 139.06 & 53.85 & 93.33 & 220.46 & 409.48 & 539.93 & 189.31 & 92.50 & 138.98 & 258.63 & 437.74 & 546.13 & 203.39 \\
\hline & 18.22 & 33.16 & 79.22 & 84.26 & 113.52 & 149.77 & 40.58 & 50.79 & 83.41 & 74.90 & 108.35 & 146.05 & 46.38 & 49.09 & 78.69 & 74.13 & 36.51 & 109.14 \\
\hline \multirow{2}{*}{ GC } & 8.62 & 31.00 & 143.28 & 354.26 & 503.20 & 132.89 & 52.96 & 95.92 & 221.44 & 404.99 & 532.24 & 189.42 & 103.76 & 147.52 & 265.99 & 444.38 & 538.33 & 211.60 \\
\hline & 16.09 & 33.70 & 79.53 & 84.56 & 114.40 & 149.56 & 41.83 & 51.80 & 81.75 & 72.50 & 105.53 & 143.95 & 59.57 & 49.91 & 75.14 & 70.16 & 44.25 & 107.65 \\
\hline \multirow{2}{*}{ CEV } & 11.22 & 31.80 & 138.44 & 354.78 & 507.09 & 131.80 & 51.71 & 93.95 & 222.38 & 413.00 & 542.20 & 190.29 & 102.79 & 146.59 & 270.51 & 459.74 & 562.75 & 214.12 \\
\hline & 16.19 & 29.58 & 77.44 & 84.28 & 116.24 & 148.92 & 39.42 & 51.35 & 84.15 & 74.29 & 106.47 & 147.32 & 60.16 & 53.31 & 78.03 & 74.47 & 48.06 & 112.39 \\
\hline \multirow{2}{*}{ HW } & 14.13 & 35.19 & 138.59 & 347.88 & 498.71 & 132.04 & 59.79 & 99.93 & 219.94 & 399.59 & 525.43 & 190.28 & 111.96 & 150.96 & 266.14 & 442.87 & 542.40 & 214.11 \\
\hline & 19.33 & 31.26 & 74.94 & 82.31 & 115.37 & 145.19 & 43.34 & 51.18 & 80.05 & 72.91 & 106.16 & 140.11 & 66.40 & 50.36 & 73.55 & 73.07 & 53.55 & 106.02 \\
\hline \multirow{2}{*}{ Heston } & 17.68 & 39.71 & 146.51 & 356.81 & 505.12 & 138.35 & 58.45 & 97.13 & 220.93 & 404.40 & 530.39 & 190.42 & 99.45 & 137.06 & 255.97 & 436.42 & 535.08 & 202.61 \\
\hline & 22.46 & 33.88 & 76.79 & 83.24 & 115.21 & 147.02 & 42.16 & 51.40 & 81.19 & 72.45 & 107.35 & 142.35 & 58.67 & 47.50 & 77.04 & 62.91 & 44.32 & 107.23 \\
\hline $\begin{array}{c}\text { No. of } \\
\text { Observations }\end{array}$ & 772 & 938 & 1697 & 414 & 222 & 4043 & 491 & 632 & 1085 & 233 & 120 & 2561 & 153 & 235 & 432 & 22 & 9 & 851 \\
\hline
\end{tabular}

Table 4(a). Moneyness Statistics of S\&P CNX Nifty 50 Index Call Options (prices in INR) for the period of Jan 1, 2008 to December 31, 2008

\begin{tabular}{cccccccc}
\hline \multirow{2}{*}{ Models } & & \multicolumn{7}{c}{ Moneyness $(\mathrm{x}=\mathrm{S} / \mathrm{K}-1)$} \\
& & DOTM & OTM & ATM & ITM & DITM & Overall \\
\hline \multirow{2}{*}{ BS } & Average & 40.44 & 72.86 & 183.08 & 369.02 & 508.98 & 161.33 \\
& Std Dev & 48.86 & 59.52 & 91.02 & 83.58 & 111.76 & 143.29 \\
& Average & 37.78 & 71.62 & 187.27 & 380.33 & 522.26 & 163.97 \\
& Std Dev & 42.12 & 57.04 & 91.75 & 84.83 & 111.17 & 146.80 \\
DVF 2 & Average & 37.15 & 70.21 & 186.93 & 380.78 & 522.21 & 163.40 \\
& Std Dev & 41.80 & 55.47 & 91.65 & 85.27 & 110.82 & 146.96 \\
\multirow{5}{*}{ GC 3 } & Average & 36.83 & 70.66 & 187.36 & 381.12 & 522.16 & 163.66 \\
& Std Dev & 40.97 & 55.85 & 91.43 & 84.38 & 111.14 & 146.91 \\
& Average & 34.28 & 68.90 & 186.16 & 374.89 & 514.03 & 161.29 \\
& Std Dev & 46.16 & 60.49 & 92.73 & 84.50 & 110.90 & 146.85 \\
& Average & 35.15 & 68.51 & 184.53 & 378.51 & 520.52 & 161.29 \\
\multirow{3}{*}{ HW } & Std Dev & 44.41 & 58.88 & 94.71 & 86.39 & 112.96 & 148.32 \\
& Average & 40.53 & 72.93 & 183.20 & 369.01 & 508.97 & 161.42 \\
\multirow{2}{*}{ Heston } & Std Dev & 48.87 & 59.51 & 91.02 & 83.58 & 111.76 & 143.27 \\
& Average & 40.65 & 72.49 & 186.34 & 376.00 & 514.52 & 163.57 \\
& Std Dev & 45.08 & 55.92 & 89.59 & 82.83 & 111.79 & 144.07 \\
& & 1416 & 1805 & 3214 & 669 & 351 & 7455 \\
\hline
\end{tabular}


Table 5. Categorical Segregation of Option Pricing Models

\begin{tabular}{|c|c|c|c|c|c|}
\hline & DOTM & ОТМ & АТМ & ITM & DITM \\
\hline $\begin{array}{l}\text { Short Maturity } \\
\qquad(\mathrm{T} \leq \mathbf{3 0})\end{array}$ & Heston & DVF & $\begin{array}{c}\text { BS, } \\
\text { DVF, } \\
\text { GC, Heston }\end{array}$ & $\begin{array}{c}\text { BS, } \\
\text { DVF, } \\
\text { CEV, Heston }\end{array}$ & $\begin{array}{c}\text { BS, } \\
\text { DVF, } \\
\text { CEV, Heston }\end{array}$ \\
\hline $\begin{array}{l}\text { Medium Maturity } \\
\qquad(30<\mathrm{T} \leq 60)\end{array}$ & $\begin{array}{l}\text { DVF, } \\
\text { GC, } \\
\text { CEV }\end{array}$ & $\begin{array}{l}\text { DVF, } \\
\text { CEV }\end{array}$ & $\begin{array}{c}\text { DVF, } \\
\text { GC, } \\
\text { CEV, } \\
\text { HW, Heston }\end{array}$ & $\begin{array}{c}\text { BS, DVF, GC CEV, } \\
\text { HW }\end{array}$ & $\begin{array}{c}\text { BS, } \\
\text { DVF, } \\
\text { GC, } \\
\text { CEV, } \\
\text { HW }\end{array}$ \\
\hline $\begin{array}{l}\text { Long Maturity } \\
\qquad(T>60)\end{array}$ & $\begin{array}{c}\text { DVF, } \\
\text { GC, } \\
\text { CEV, Heston }\end{array}$ & DVF, Heston & Heston & $\begin{array}{c}\text { BS, } \\
\text { DVF, } \\
\text { GC, } \\
\text { HW, } \\
\text { Heston, }\end{array}$ & Heston \\
\hline
\end{tabular}

